\title{
Pneumonia Detection Using Convolutional Neural Networks
}

\author{
Puneet Gupta
}

Information Technology, Maharaja Agrasen Institute of Technology Delhi, India

To Cite this Article

Puneet Gupta, "Pneumonia Detection Using Convolutional Neural Networks", International Journal for Modern Trends in Science and Technology, Vol. 07, Issue 01, January 2021, pp.- 77-80.

\section{Article Info}

Received on 22-November-2020, Revised on 18-December-2020, Accepted on 28-December-2020, Published on 04-January-2021.

\section{ABSTRACT}

Abstract-Pneumonia is a life-threatening infectious disease affecting one or both lungs in humans commonly caused by bacteria called Streptococcus pneumoniae. One in three deaths in India is caused due to pneumonia as reported by World Health Organization (WHO). Chest X-Rays which are used to diagnose pneumonia, need expert radiotherapists for evaluation. Thus, developing an automatic system for detecting pneumonia would be beneficial for treating the disease without any delay particularly in remote areas. Due to the success of deep learning algorithms in analyzing medical images, Convolutional Neural Networks (CNNs) have gained much attention for disease classification. In addition, features learned by pre-trained CNN models on large-scale datasets are much useful in image classification tasks. In this work, we appraise the functionality of pre-trained CNN models utilized as feature-extractors followed by different classifiers for the classification of abnormal and normal chest X-Rays. We analytically determine the optimal CNN model for the purpose. Statistical results obtained demonstrates that pretrained CNN models employed along with supervised classifier algorithms can be very beneficial in analyzing chest X-ray images, specifically to detect Pneumonia. In this project Transfer learning and a CNN Model is used to detect whether the person has pneumonia or not using chest $x$-ray.

KEYWORDS: Pneumonia, Convolutional Neural Networks, Detection, Deep Leaning, Transfer Learning.

\section{INTRODUCTION}

PNEUMONIA is an illness that disturbs the lung air sacs of an infected person. It is triggered by bacteria, fungi, or a virus that infects the air sacs of lungs that fill up with discharge fluids that leads to chills, fever, coughing with mucus, and breathing trouble among persons diagnosed with this disease. Children below five years of age and elderly patients with weak immune system are vulnerable to this type of diseases. Pneumonia has killed over a million children worldwide in 2018 and remains a life-threatening disease now a days if not detected or diagnose earlier. Radiography,
CT-scan, or MRI is the common method to discover pneumonia. Medical personnel check the patient's radiograph of their chest to determine if they are infected with pneumonia or not. In addition, the usual method for finding pneumonia is through medical history and laboratory results of the patient.

Radiograph of chest is penetrated through $\mathrm{X}$-rays where the soft tissues produces a dark color and hard tissues like bones produces a bright color. Patients diagnosed with pneumonia shows the chest cavity signs of fluids filling the air sacs of lungs as for the radiograph picture appears 
brighter. Several abnormalities may be seen on lung cavities as brighter color may represent such as cancer cells, blood vessels swelling, and abnormality of heart. To validate the range and spot of an infected area of the lungs, chest $\mathrm{x}$-rays is the utmost method. In these method, emergence of the disease can be imprecise and misinterpreted with another illness. Therefore, the undertaking is pleasing in the improvement of the processing in medical situations in isolated areas for pneumonia detection. The researchers were able to train and assessed CNN model's performance and classify chest $\mathrm{x}$-rays with normal and infected with disease using different classifiers. With the recent development of Computer Aided Design (CAD) tools becomes the most important field of research in artificial intelligence and machine learning. CAD systems has proven in facilitating the medical field such as breast cancer detection, classification of disease using mammograms, lung cancer detection, etc. CAD system is an applicable instrument in use today for diagnosis and classification of diseases in medical imaging. In achieving the precise diagnosis, the medical personnel integrate the CAD to assist and verify to support their decision making. Significant features of the images are valuable in employing machine learning techniques in this system compared to the traditional handcrafted features which has limitations in extracting significant features. The progress in a more intelligent future is now productive through generations. This technological improvement today reached new step closer in human intelligence. The deep learning has gained the ability in simulating the function of the human brain. It recommends the solution to solve real-life problems. The deep learning by means of the convolutional neural networks has ability in obtaining significant characteristics in image classification tasks and provides medical promising results in image analysis . CNN advantagesis capable in assisting the identification of some features from an image and use this feature to generate probabilities in classifying specific input. The contribution of this study is developed an optimized deep learning models of CNN that can detect and classify pneumonia diseases efficiently [10]. The work consists of an optimized CNN models and experimental analysis of each model towards the detection and classification of pneumonia diseases.This research article consists of the following sections: introduction, convolutional neural network, methodology, experimental results and discussion, conclusion and recommendations, acknowledgments and references.

\section{STRUCTURE OF PAPER}

The paper is organized as follows: In Section 1, the introduction of the paper is provided along with the structure, important terms, objectives andoverall description. In Section 2 we discuss related work. In Section 3 we have the complete information about Convolution Neural Network and in section 4 we haveMethodology and the process description.

Section 5 tells us about the future scopeand concludes the paper with acknowledgement andreferences.

\section{OBJECTIVES}

Different models of deep learning and transfer learning are analyzed in this work for the image classification application. Using Deep Learning features are extracted from the images and are used for classification of $\mathrm{x}$-rays having pneumonia. A convolutional neural network architecture is made and it is trained with the images of $\mathrm{x}$-rays with pneumonia and normal $\mathrm{x}$-rays which will be used for further classification. We also used Vgg16 and Vgg19 transfer learning technique to compare the accuracy of the models. CNN models have been created from scratch and trained on Chest X-Ray Images (Pneumonia) dataset on Kaggle.

\section{RELATED WORK}

CNN is a very powerful algorithm which is widely used for image classification and object detection. The hierarchical structure and powerful feature extraction capabilities from an image makes $\mathrm{CNN}$ a very robust algorithm for various image and object recognition tasks. There are various architectures of CNNs available which have been key in building algorithms which power and shall power AI as a whole in the foreseeable future. Some of them are: LeNet, AlexNet, VGGNet, GoogLeNet, ResNet, ZFNet and etc

\section{Convolution NeURal Network}

Process of CNN is to detect and categorize images from learned features. It is very effective in a multi-layered structure when obtaining and assessing necessary features of graphical images. Figure 1 illustrates the CNN process. 
Pneumonia Detection using Convolutional Neural Network (CNN)

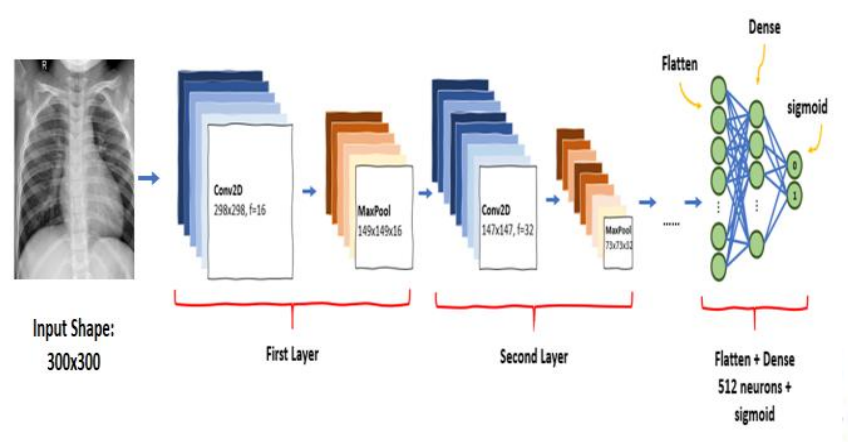

\subsection{Convolution Layer}

Convolution in CNN is performed on an input image using a filter or a kernel. To understand filtering and convolution you will have to scan the screen starting from top left to right and moving down a bit after covering the width of the screen and repeating the same process until you are done scanning the whole screen.

\begin{tabular}{|c|c|c|c|c|c|c|c|}
\hline $1_{\times 1}$ & $1_{\times 0}$ & $1_{\times 1}$ & 0 & 0 \\
\hline $0_{\times 0}$ & $1_{\times 1}$ & $1_{\times 0}$ & 1 & 0 \\
\hline $0_{\times 1}$ & $0_{\times 0}$ & $1_{\times 1}$ & 1 & 1 \\
\hline 0 & 0 & 1 & 1 & 0 \\
\hline 0 & 1 & 1 & 0 & 0 \\
\hline
\end{tabular}

Fig. 2 Demonstration of Convolution Layer

\subsection{Max-Pooling Layer}

This layer cut down the values further to half of its original value by choosing only max values from the kernel matrix. The sample illustration is the $4 \times 4$ matrix pixel values of an input image degraded into $2 \times 2$ filters. Its process is described in Fig.

Single depth slice

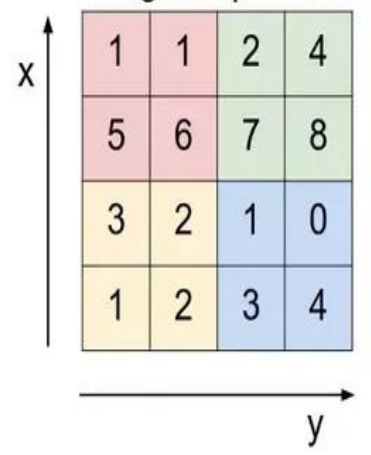

max pool with $2 \times 2$ filters and stride 2

Fig. 3 Mlustration of Max Pooling Layer

\subsection{Transfer Learning.}

For an effective classification, the models are set for proper setting to the needed task. Transfer learning let the model save to original parameter settings of the previously trained model to bring effective score without using intense computing power [12]. The Fine-Tuning method can extract new features from pneumonia and change the original 1000 neurons for example the VGGNet model to only four [13]. Also, FC or dense layers of 4096 down to a more significant number of 512 and 256 to prevent any waste of computing resources. Hyperparameters facilitate the deep learning model to produce significant results just as the actual training procedure takes place. Selecting the exact values can initially make a huge dissimilarity by making hyperparameter tuning a necessary process. Still, there are no precise hyperparameters for every algorithm [14]. Thus, a practical experimentation must take place.

\subsection{Classification}

Every single block as shown in Fig. 1 are used in architecture followed by wholly inter-connected layers and softmax activation. Feature extraction is composed of an input image, convolution, max-pooling while classification involves fully interconnected layers and output .

\section{METODODLOGY:}

Data augmentation: is a common step used for increasing the dataset size and the model generalizability. Essentially, it is the process of artificially increasing the size of a dataset via transformations - rotation, flipping, cropping, stretching, lens correction

Feature Extraction: In this part, the network will perform a series of convolutions and pooling operations

duringwhichthefeaturesaredetected.Ifyouhadap ictureofazebra,thisisthepartwhere the network would recognize its stripes, two ears, and fourlegs.

Classification: Here, the fully connected layers will serve as a classifier on top of these extracted features. Theywillassignaprobabilityfortheobjectontheim agebeingwhatthealgorithmpredicts itis. 


\section{FUTURE SCOPE AND CONCLUSION}

We have assessed the performance of $3 \mathrm{CNN}$ models namely;

VGG16, VGG19 and a CNN model that we built from scratch

For VGG16 we got validation accuracy of $92 \%$ and training accuracy of about $97 \%$ and for VGG19 we got validation accuracy of $89 \%$ and training accuracy of about $95 \%$

And for the model that we built we got validation accuracy of $93 \%$ and training accuracy of about 99\%.

Pneumonia dataset used in the study. Table 2 shows the overall performance of 3 models used in thisstudy.

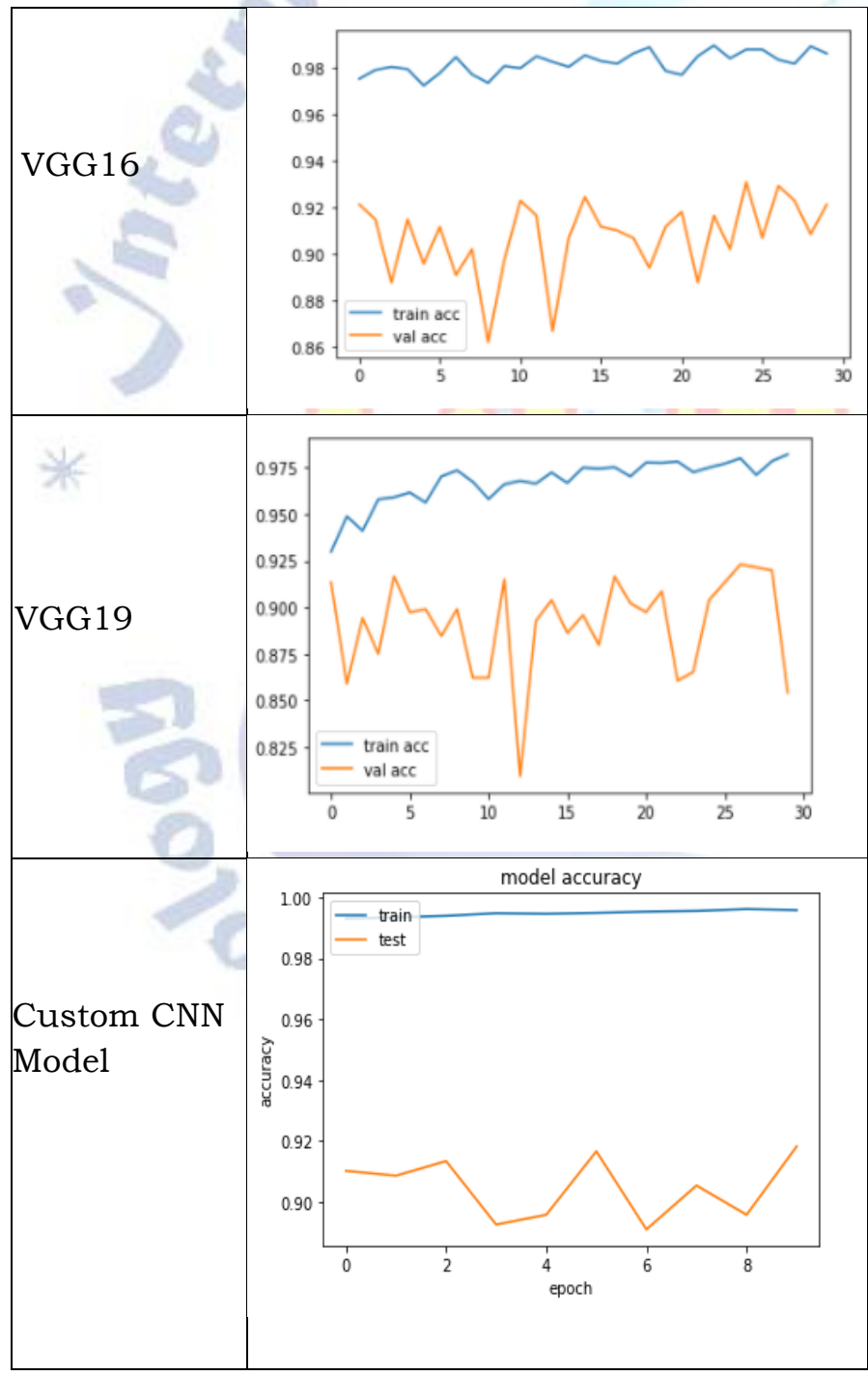

Table 1. Result of Training and Validation of Five CNN models

This research explores the deep learning function in detecting pneumonia through computer vision using three convolutional neural network models. Every CNN models identified are assessed physically so that the framework in the feature extractionis employed. The pneumonia infected diseases and normal chest $\mathrm{x}$-ray image dataset are acquired. Our study enables to identify among the three models the best model to detect pneumonia. All the models have performed well on detecting pneumonia and normal chest x-rays. We have demonstrated how to classify positive and negative pneumonia data from a collection of X-ray images. We build our model from scratch, which separates it from other methods that rely heavily on transfer learning approach. We also used transfer learning approach to solve and we got good results with the CNN model we built and it was more accurate than the transfer learning models that we used.

In the future, this work will be extended to detect and classify X-ray images consisting of Covid-19 and pneumonia. Distinguishing X-ray images that contain Covid-19 and pneumonia has been a big issue in recent times, and our next approach will tackle this problem

\section{ACKNOWLEDGEMENT}

Aside from God Almighty, the researchers would like to express their gratitude to their families, relatives, and dear ones whose involvement made a significant impact on the accomplishment of this task.

\section{REFERENCES}

[1] https://medium.com/@dipti.rohan.pawar/improving-perf ormance-of-convolutional-neural-network-2ecfe0207de7E.

[2] https://arxiv.org/abs/1409.1556

[3] https://machinelearningmastery.com/how-to-use-transfe r-learning-when-developing-convolutional-neural-network -models/

[4] https://analyticsindiamag.com/practical-comparison-of-t ransfer-learning-models-in-multi-class-image-classificatio $\mathrm{n} /$

[5] https://medium.com/abraia/first-steps-with-transfer-lear ning-for-custom-image-classification-with-keras-b941601f cad5 